\title{
Análisis de Capacidades de Gestión del Conocimiento para la Competitividad de PYMES en Colombia
}

\author{
Albeiro Hernández ${ }^{(1)}$, Carlos E Marulanda ${ }^{(2)}$ y Marcelo López ${ }^{(3)}$ \\ (1) Universidad Cooperativa Colombia sede Pereira, Centro de investigaciones. La Julita, Pereira- \\ Colombia. (e-mail: albeiro.hernandez@ucc.edu.co) \\ (2) Universidad Nacional de Colombia, Facultad de Administración, Departamento de Administración. \\ Campus Palogrande. Calle 27 No. 64-60, Manizales-Colombia y Universidad de Caldas, Facultad de \\ Ingeniería, Departamento de Sistemas e Informática, Calle 65 No. 26-10, Manizales-Colombia. \\ (e-mail: cemarulandae@unal.edu.co) \\ (3) Universidad de Caldas, Facultad de Ingeniería, Departamento de Sistemas e Informática, Calle 65 \\ No.26-10, Manizales-Colombia. (e-mail: mlopez@ucaldas.edu.co)
}

Recibido Jul. 22, 2013; Aceptado Sep. 23, 2013; Versión final recibida Sep. 30, 2013

\section{Resumen}

Se analiza las capacidades de gestión del conocimiento para la competitividad de Pequeñas y Medianas Empresas (PYMES) de Colombia. Se evaluaron trecientas veintiuna empresas en relación al análisis organizacional, los procesos y las herramientas de tecnología de información y las comunicaciones, para gestionar conocimiento. Se evidencian avances en los procesos para la gestión del conocimiento, pero existe un lento avance en el desarrollo organizacional y en el uso de tecnologías de la información y las comunicaciones. Este estudio aporta a la comunidad académica y a las pequeñas y medianas empresas del país con una línea base del estado en gestión del conocimiento, de manera que se puedan formular acciones para potenciar su competitividad.

Palabras clave: gestión de conocimiento, análisis organizacional, PYMES, TICs,

\section{Analysis of Knowledge Management Capabilities for Competitiveness of SMEs in Colombia}

\begin{abstract}
The capabilities of knowledge management for competitiveness of Small and Medium Size Enterprises (SME) in Colombia is analyzed. For this, three hundred twenty one companies were evaluated in relation to organizational analysis, processes and tools of information and communication technology, to manage knowledge. Advances are evident in the processes of knowledge management, but there is slow progress in organizational development and the use of information and communication technology. This study contributes to the academic community and to the small and medium size companies in the country with a baseline of the state of knowledge management, so that they can formulate actions to enhance their competitiveness.
\end{abstract}




\section{INTRODUCCIÓN}

Colombia define la PYME según sus activos totales en salarios mínimos mensuales vigentes y el número de empleados (Ley 905 de 2004). Una empresa mediana de 51 a 200 empleados y activos entre5.001 y15.000, empresa pequeña de 11 a 50 y activos entre501 y5.000 y microempresa hasta 10 empleados y activos inferiores a 500. Se estima que existen más de un millón de micros y pequeñas empresas que contribuyen a la producción nacional con una cifra superior al $50 \%$ y generan más del $70 \%$ del empleo, en los sectores de industria, comercio y servicios. Del total, 300.000 son formales, de las cuales el $75 \%$ son microempresas, $24 \%$ PYMES y $1 \%$ gran empresa. Su distribución en diferentes áreas se concentra en el comercio, con un $54,66 \%$, servicios con un $31,60 \%$, industria con un $12,22 \%$ y otros con un $1,52 \%$ (Cala, 2005).

Los principales obstáculos de las PYMES para su desarrollo en Colombia, según (Sánchez, et al., 2007), son: las restricciones al crédito, las dificultades en la identificación y acceso a la tecnología adecuada, la formalización y absorción de nuevas tecnologías, las limitaciones técnicas y competitivas que imponen las escalas de producción, la deficiente infraestructura física, la falta de asociatividad empresarial, la carencia de directivos con capacidad gerencial y pensamiento estratégico y la dificultad de cimentar la articulación del sector con la gran empresa y con los sistemas de compras estatales. Varios autores (Benavides, 1998; Bueno, 2003a; Díaz et al., 2005; Ampudia, 2006) coinciden en la necesidad que las PYMES se integren en redes, conglomerados o clúster para solucionar los problemas de competitividad, la cual según (Porter, 1991): "se basa en la productividad con la cual esta produce bienes y servicios. Está fundamentada en las bases microeconómicas de una nación: la sofisticación de las operaciones y estrategias de una compañía y la calidad del ambiente microeconómico de los negocios en la cual las compañías compiten".

Las empresas tienen la exigencia de generar y fortalecer las ventajas competitivas sostenibles y sustentables para responder, adaptar y sostenerse (Porter, 1991;Alvarez, 2004; OCDE, 1997; Matilla y Chalmeta, 2007)y lo han hecho basados en diversos enfoques, que van desde la gestión del conocimiento, la incorporación a un clúster (Morales et al., 2010), la innovación y las TIC (Romero et al., 2009),(Domínguez et al., 2007), (Cuenca et al., 2008),y el mejoramiento continuo (Meisel et al., 2006) entre otros. El enfoque de la innovación debe ser parte vital de todos los sectores económicos y hacerse extensivo a todos sus eslabonamientos (DNP, 2005; EUROSTAT, 2006; Morales et al., 2010) y en el marco de la gestión del conocimiento es un agente que dinamiza externalidades en los sistemas productivos, (Hautala, 2011; Knudsen, 1995; Lopez, et al., 2011; López y Calderón, 2006), además de ser un factor fundamental de la competitividad.

El enfoque de gestión del conocimiento parte de un proceso que continuamente asegura el desarrollo y aplicación de todo tipo de conocimientos pertinentes en una empresa, con el objeto de mejorar su capacidad de resolución de problemas y así contribuir a la sostenibilidad de sus ventajas competitivas (Andreu y Siever, 1999).Alavy y Leidner (2001), definen gestión de conocimiento en organizaciones, como aquella que se refiere a la identificación y el aprovechamiento del conocimiento colectivo en una organización para ayudar a la organización competir, en el marco de cuatro procesos básicos de creación, almacenamiento y recuperación, transferencia y aplicación del conocimiento. Davenport y Prusak (1998), plantean que el conocimiento es una mezcla de flujo de experiencias enmarcadas, valores, información contextual que proporciona un marco para evaluar e incorporar nuevas experiencias e información. Se origina y se aplica en la mente de los conocedores. En las organizaciones, a menudo se manifiesta no sólo en los documentos o depósitos, sino también en las rutinas organizativas, procesos, prácticas y normas. Su evaluación (Muñoz y Muñoz, 2010), según Marr (2004) se basa en el capital intelectual (Cl), el cual es ampliamente reconocido como la fuente fundamental de la verdadera y una ventaja competitiva sostenible. El conocimiento es la base del $\mathrm{Cl}$ y es por lo tanto, en el centro de las capacidades de organización, las cuales se pueden clasificar en: las relaciones, de las partes interesadas que incluyen todas las formas de las relaciones de una empresa con sus grupos de interés; los recursos humanos, que abarca los activos de conocimiento proporcionados por los empleados; la Infraestructura física, que incorpora todos los activos de infraestructura; la cultura, que abarca valores organizacionales y la filosofía de gestión; las prácticas y rutinas, que incluyen las prácticas internas formales e informales y la propiedad intelectual, que es la suma de los activos de conocimiento.

Se han hecho algunos estudios relacionados, como es el caso de las tesis doctorales realizadas en España: la caracterización del conocimiento en PYMES que realizan proyectos TIC: un modelo de análisis y valoración(Lobato, 2008) y el desarrollo de un modelo de gestión del conocimiento en la cadena de suministro de la industria agroalimentaria(Martínez, 2011).En Colombia se observan tendencias de acuerdo a los resultados de investigaciones relacionadas con: el grado de coincidencia entre el concepto y el enfoque que tienen las organizaciones sobre la identificación del aprendizaje organizacional y la generación de capital intelectual en la gestión del conocimiento(Carrillo, 2005; Garzón, 2006; Paniagua, et al., 2007), la gestión del conocimiento (Briceño y Bernal, 2010),la generación de valor y el fortalecimiento de las ventajas 
competitivas de las compañías (Bernal et al., 2010) y la valoración de la sociedad del conocimiento (López, et al., 2011). Dichos estudios reconocen la capacidad de desarrollo delas empresa para satisfacer las necesidades de los clientes locales, regionales y nacionales, pero con la gestión del conocimiento serían mejores los resultados, tal como lo establecen (Martinez, 2006; Kok, 2007; Shin et al., 2001; Johannessen et al., 2005; Nahapiet y Ghoshal, 1998).

Para este caso se presentan los resultados de la aplicación de un modelo de valoración de la gestión del conocimiento para empresas, mediante una evaluación integral y permanente de las siguientes categorías: rasgos organizacionales, competencias, prácticas, procesos y tecnologías de información y comunicaciones TI, en 321 PYMES de las ciudades de Armenia, Barranquilla, Bogotá, Bucaramanga, Cali, Manizales, Medellín y Pereira de Colombia.

\section{MATERIALES Y MÉTODOS}

Para el desarrollo, se utilizó una investigación exploratoria, para analizar e identificar las principales variables del modelo de evaluación, una investigación descriptiva, para conocer las situaciones predominantes con la representación exacta de los procesos, una investigación de tipo correlacional para encontrar la correlación entre las variables.

\section{Muestreo}

Se aplicó la encuesta a través de internet a una muestra de la población, 321PYMES de diversas regiones de Colombia. Previa la valoración, se desarrolló una prueba piloto de la encuesta con expertos sobre el tema, además de una revisión con algunos de los actores académicos y del ámbito productivo. Para la estructuración del cuestionario se formularon preguntas en escala Likert, las cuales se calificaron de 1 a 5 , donde 1 , no realizado, 2 , realizado parcialmente, 3 , realizado en intervalos de tiempo, 4 , realizado con regularidad y 5 realizado completamente.

\section{Categorías evaluadas}

Las categorías y variables se fundamentan en los modelos y planteamientos desde la perspectiva europea, (CEN, 2004), el foro europeo de conocimiento, (Chen, 2008), y los desarrollos de autores como (Bueno, 2003b), (DelMoral et al., 2007) y (López, 2010), en los que se define la medición desde el capital humano, organizativo, tecnológico, relacional e intangible, entre otros, considerando las interrelaciones y las perspectivas dinámicas de una organización del conocimiento, por medio de métricas, herramientas e indicadores específicos, ligados a las competencias individuales y las capacidades organizacionales. Estas se agruparon tal como se observa en la tabla 1:

Tabla 1: Categorías y variables a evaluar

\begin{tabular}{|l|l|r|}
\hline \multicolumn{1}{|c|}{ Categorías } & \multicolumn{1}{|c|}{ Variables } & $\begin{array}{c}\text { № de } \\
\text { preguntas }\end{array}$ \\
\hline $\begin{array}{l}\text { Rasgos } \\
\text { Organizacionales }\end{array}$ & $\begin{array}{l}\text { Tipo de organización, capital intelectual, estímulos, direccionamiento estratégico y } \\
\text { apropiación de conocimiento. }\end{array}$ & 27 \\
\hline Procesos & $\begin{array}{l}\text { consideraciones generales, procesos para identificar, generar, retener, compartir y } \\
\text { aplicar conocimiento }\end{array}$ & 52 \\
\hline TIC & $\begin{array}{l}\text { Web social y semántica para identificar, generar, retener, compartir y aplicar } \\
\text { conocimiento; TIC para el modelo SECl (socializar, exteriorizar, combinar e } \\
\text { interiorizar conocimiento) de (Nonaka \& Takeuchi, 1999); TIC para un modelo } \\
\text { holístico de gestión de conocimiento. }\end{array}$ & 61 \\
\hline
\end{tabular}

Para revisión de la herramienta de encuesta y los resultados, ingresar al portalwww.logopoliskpo.com/sigeco, con el usuario: carloselog@gmail.com y la contraseña: carlose1999. Además de considerar las áreas de competitividad establecidas por el consejo privado de competitividad colombiano.

\section{RESULTADOS Y DISCUSIÓN}

El proceso de recolección de datos se hizo personal y telefónicamente con representantes de las empresas en el momento del registro, considerando que tuvieran comprensión de procesos relacionados con la gestión del conocimiento organizacional y la competitividad, además de hacer un muestreo aleatorio de comprobación. Se presenta a su vez la matriz de correlación, ver tabla 2, la cual enseña un alto grado de 
correlación entre las categorías evaluadas, lo que hace posible aplicar el método de componentes principales.

Tabla 2: Tabla de correlaciones (valor $p$ )

\begin{tabular}{|c|c|c|c|c|c|c|c|c|c|c|c|c|c|c|c|c|c|c|c|}
\hline Varia & $\begin{array}{l}\text { D } \\
\frac{D}{8} \\
\mathbb{D} \\
\mathbb{D}\end{array}$ & $\begin{array}{l}\overline{\bar{\alpha}} \\
\stackrel{\bar{D}}{\vec{Q}} \\
\overline{\bar{d}}\end{array}$ & 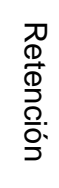 & 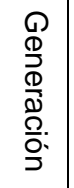 & 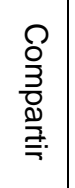 & $\begin{array}{l}\frac{D}{0} \\
\overline{\bar{D}} \\
\stackrel{D}{D} \\
\bar{D}:\end{array}$ & $\begin{array}{l}\overline{\bar{z}} \\
0 \\
0 \\
0 \\
0 \\
0\end{array}$ & $\subseteq$ & 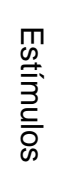 & 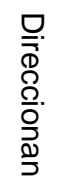 & $\begin{array}{l}\frac{3}{0} \\
\text { 응 } \\
\frac{0}{0} . \\
\frac{0}{0} \\
\text { 음: }\end{array}$ & 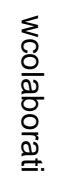 & 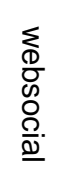 & 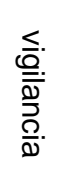 & $\begin{array}{l}\frac{O}{\bar{D}} . \\
\frac{\Phi}{0} \\
\frac{0}{0} \\
\overline{0}\end{array}$ & 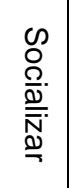 & 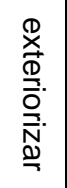 & 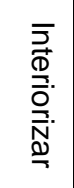 & 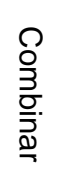 \\
\hline Aspgen & & ,03 & 84 & ,95 & ,84 & ,14 & ,17 & ,26 & , 17 & ,11 & , 11 & ,07 & ,61 & ,02 & ,00 & ,23 & ,21 & ,22 & ,27 \\
\hline Identificació & , 03 & & ,25 & ,93 & ,64 & ,67 & ,05 & , 10 & ,02 & , 00 & ,00 & ,01 & , 18 & 01 & , 00 & 24 & ,26 & ,25 & ,25 \\
\hline Retención & ,84 & ,25 & & ,97 & ,26 & ,23 & ,18 & 21 & ,12 & ,20 & ,16 &, 51 & ,17 & ,28 & ,65 & \begin{tabular}{|l|}
, 78 \\
\end{tabular} & ,84 & ,78 & ,80 \\
\hline Generación & ,95 & ,93 & ,97 & & ,08 & ,62 & ,38 & ,35 & ,42 & 66 & 61 & ,71 & ,76 & 89 & ,98 & ,14 & , 11 & ,12 & ,11 \\
\hline Com & 4 & ,64 & ,26 & ,08 & & ,87 & ,08 & ,06 & ,95 & ,33 & ,72 & ,87 & ,57 & ,49 & ,82 & \begin{tabular}{|l|}
, 15 \\
\end{tabular} & , 15 & ,13 & ,19 \\
\hline Aplicación & ,14 & ,67 & ,23 & ,62 & ,87 & & ,79 & ,90 & ,87 & ,79 & ,96 &, 72 & ,41 & ,47 & ,33 & \begin{tabular}{|l|}
, 38 \\
\end{tabular} & ,28 & ,34 & ,37 \\
\hline Tiposorg & , 17 & ,05 & ,18 & ,38 & ,08 & ,79 & & ,00 & ,26 & ,00 & ,09 &, 12 & , 17 & ,07 & , 10 & \begin{tabular}{|l|l|}
, 03 \\
\end{tabular} & ,04 & ,03 & ,05 \\
\hline $\mathrm{Cl}$ & ,26 & , 10 & ,21 & ,35 & ,06 & 90 & ,00 & & ,36 & ,01 & , 13 & , 13 & ,12 & , 15 & , 15 & \begin{tabular}{|l|}
, 02 \\
\end{tabular} & ,04 & ,02 & ,04 \\
\hline Estímulos & ,17 & ,02 & ,12 & ,42 & ,95 & ,87 & ,26 & ,36 & & ,11 & ,00 & , 10 & ,22 & ,04 &, 12 & \begin{tabular}{|l|}
, 82 \\
\end{tabular} & ,86 & 83 & ,84 \\
\hline Direccionan & ,11 &, 00 & ,20 & ,66 & ,33 & ,79 & ,00 & 01 & , 11 & & ,02 & ,03 &, 10 & ,05 & ,02 & \begin{tabular}{|l|}
, 08 \\
\end{tabular} & , 10 & ,08 & ,08 \\
\hline Apropiación & , 11 & ,00 & ,16 & ,61 & ,72 & ,96 & ,09 & ,13 & , 00 & ,02 & & ,01 & ,06 & ,05 & ,03 & ,41 & ,48 & ,44 & ,46 \\
\hline wcolaborativ & ,07 & 01 &, 51 & ,71 & ,87 & ,72 & ,12 & ,13 & , 10 & ,03 & ,01 & & ,06 & ,15 &, 00 & ,22 & ,29 & ,25 & ,26 \\
\hline websocial & ,61 & ,18 & ,17 & ,76 & ,57 & ,41 & ,17 & ,12 & ,22 &, 10 & ,06 & ,06 & &, 52 & ,28 & \begin{tabular}{|l|}
, 41 \\
\end{tabular} &, 56 & ,47 & ,47 \\
\hline vigilancia & ,02 & 01 & ,28 & ,89 & ,49 & ,47 & ,07 & , 15 & ,04 & ,05 & ,05 & , 15 & ,52 & & ,04 & ,30 & ,27 & ,28 & ,32 \\
\hline ciberaplic & 00 &, 00 & ,65 & ,98 & ,82 & ,33 & ,10 & ,15 &, 12 & ,02 & ,03 & ,00 & ,28 & ,04 & & , 14 & ,15 &, 15 & ,15 \\
\hline socializar & ,23 & ,24 & ,78 & ,14 & ,15 & ,38 & ,03 & ,02 & ,82 & ,08 & ,41 & ,22 & ,41 & ,30 & ,14 & & , 00 & ,00 & ,00 \\
\hline exteriorizar & ,21 & ,26 & 84 & ,11 & ,15 & ,28 & ,04 & ,04 & 86 &, 10 & ,48 & ,29 & ,56 & ,27 & , 15 & \begin{tabular}{|l|}
, 00 \\
\end{tabular} & & ,00 & ,00 \\
\hline Interiorizar & ,22 & ,25 & ,78 & ,12 & ,13 & ,34 & ,03 & ,02 & ,83 & ,08 & ,44 & ,25 & ,47 & ,28 & , 15 & , 00 & ,00 & & ,00 \\
\hline Combinar & ,27 & ,25 & ,80 & ,11 & ,19 & ,37 & ,05 & ,04 & 84 & ,08 & ,46 & ,26 & ,47 & ,32 &, 15 & ,00 & ,00 & ,000 & \\
\hline
\end{tabular}

Desde la categoría rasgos organizacionales para la gestión del conocimiento, los resultados se graficaron tal como se observa en la figura 1.

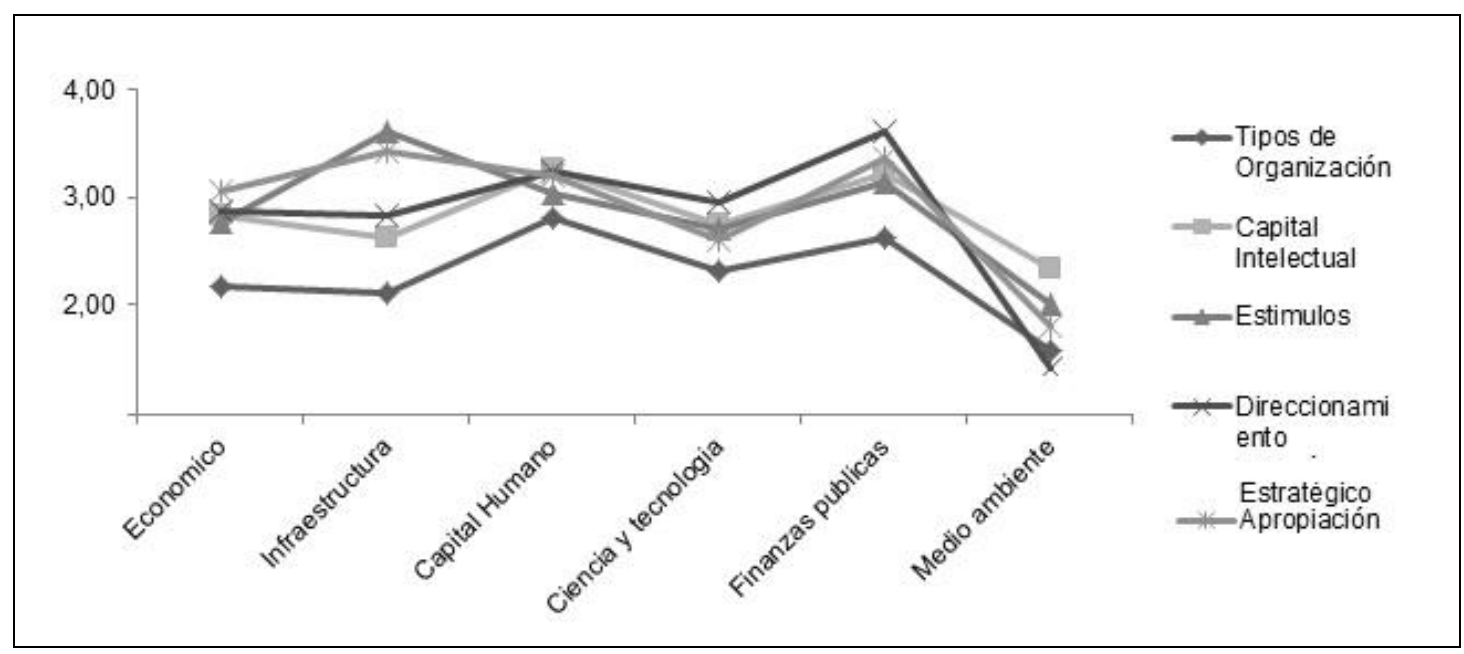

Fig. 1: Rasgos organizacionales para la gestión del conocimiento

De acuerdo a la escala de calificación, el promedio de las variables evaluadas está alrededor de 2.7, lo que muestra una calificación baja en los rasgos organizacionales para gestionar conocimiento en los diversos sectores competitivos de las PYMES de Colombia. Se destacan valores por debajo del promedio en el sector medio ambiente, desde los tipos de organización, el capital intelectual, los estímulos, el direccionamiento estratégico y la apropiación de la gestión del conocimiento, lo que muestra pocos 
esfuerzos para gestionar el conocimiento en este sector competitivo. Lo que hace necesario investigar con mayor profundidad en los sectores a fin de establecer estrategias para su mejoramiento.

Mientras se cuenta con resultados por encima del promedio en los estímulos para gestionar conocimiento en el sector de infraestructura, el direccionamiento estratégico para gestionar conocimiento en los sectores de ciencia y tecnología y finanzas públicas.

Desde la categoría procesos para la gestión del conocimiento, los resultados se graficaron tal como se observa en la figura 2.

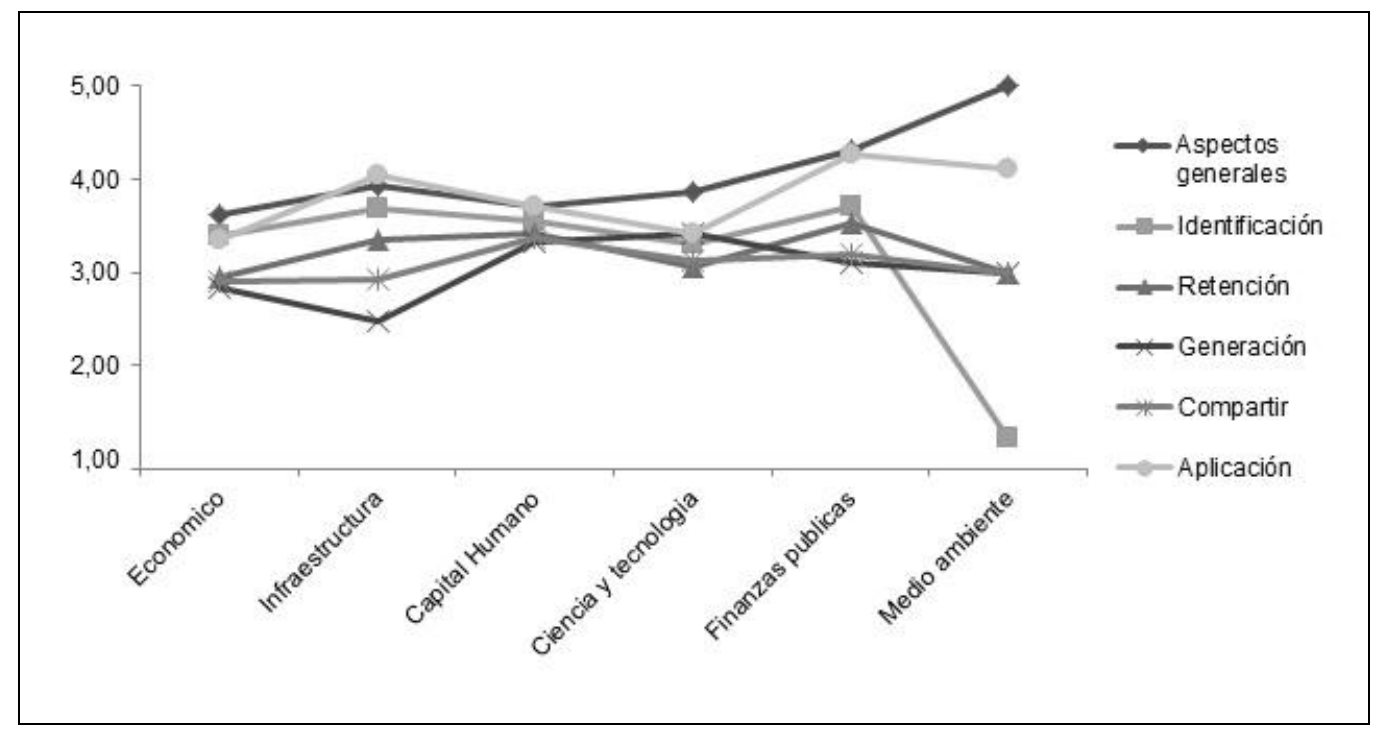

Fig. 2: Procesos para la gestión del conocimiento

De acuerdo a la escala de calificación, el promedio de las variables evaluadas está alrededor de 3.4, lo que muestra una calificación promedia en los procesos organizacionales para gestionar conocimiento en los diversos sectores competitivos de las PYMES. Se destacan valores por debajo del promedio en el sector medio ambiente, desde la identificación del conocimiento y en el sector de infraestructura desde la generación de conocimiento, lo que muestra pocos esfuerzos para gestionar el conocimiento en estos sectores competitivos. Lo que hace necesario investigar con mayor profundidad en los sectores a fin de establecer estrategias para su mejoramiento.

Mientras se cuenta con resultados por encima del promedio en los aspectos generales y la aplicación de la gestión de conocimiento en el sector medio ambiente y los sectores de finanzas públicas y de ciencia y tecnología; la aplicación de la gestión del conocimiento en los sectores de finanzas públicas e infraestructura y la identificación de conocimiento en los sectores de finanzas públicas e infraestructura.

Desde la categoría TI para la gestión del conocimiento, los resultados se graficaron tal como se observa en la figura 3.

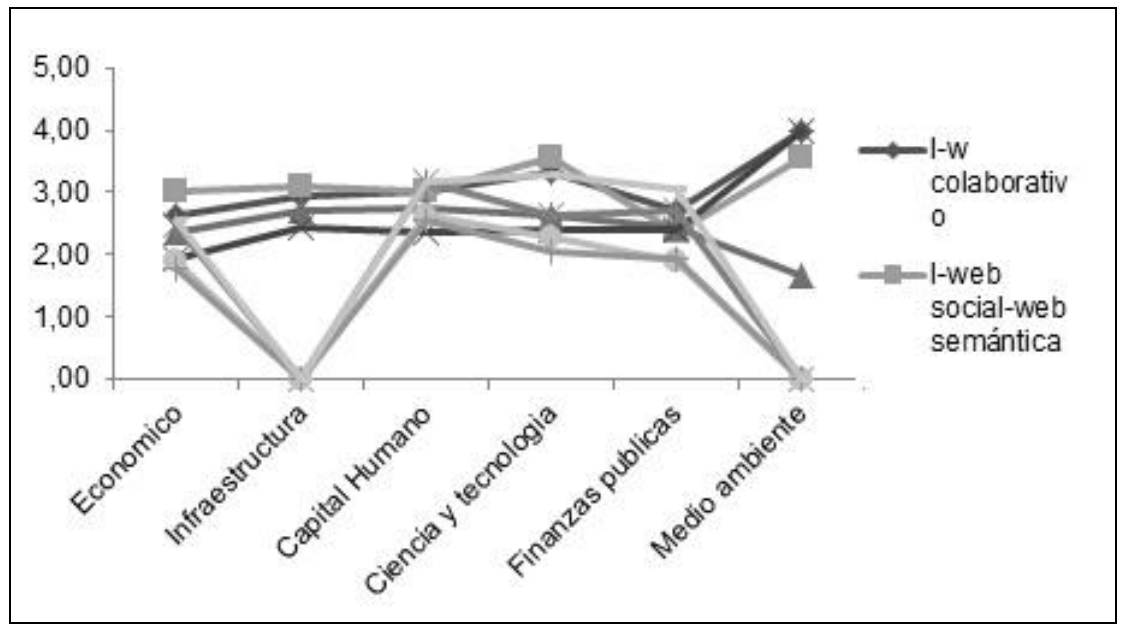

Fig. 3: Tecnologías de información y comunicaciones para la gestión del conocimiento 
De acuerdo a la escala de calificación, el promedio de las variables evaluadas está alrededor de 2.2, lo que muestra una calificación baja en el uso de TI para gestionar conocimiento en los diversos sectores competitivos de las PYMES. Se destacan valores por debajo del promedio en los sectores de medio ambiente e infraestructura, desde combinar, exteriorizar y socializar el conocimiento, además de la vigilancia en ciencia tecnológica. Lo que hace necesario investigar con mayor profundidad en los sectores a fin de establecer estrategias para su mejoramiento.

Mientras se cuenta con resultados por encima del promedio en el uso de ciber-aplicaciones y de web social y web semántica en el sector medio ambiente; así como el uso de web social y web semántica y el trabajo colaborativo en el sector de ciencia y tecnología. Dada la cantidad de variables que se evaluaron, se realizó un análisis de componentes principales, con el software estadístico SPSS para determinar, cuáles de ellas explican la gestión del conocimiento en los sectores de competitividad del país, ver tabla 3.

Tabla 3: Componentes principales

\begin{tabular}{|c|c|c|c|c|c|c|}
\hline \multirow[t]{2}{*}{ Componente } & \multicolumn{3}{|c|}{ Autovalores iniciales } & \multicolumn{3}{|c|}{ Sumas de las saturaciones al cuadrado } \\
\hline & Total & $\%$ de la varianza & $\%$ acumulado & Total & $\%$ de la varianza & $\%$ acumulado \\
\hline 1 & 11,328 & 59,621 & 59,621 & 11,328 & 59,621 & 59,621 \\
\hline 2 & 3,969 & 20,889 & 80,51 & 3,969 & 20,889 & 80,51 \\
\hline 3 & 2,514 & 13,234 & 93,744 & 2,514 & 13,234 & 93,744 \\
\hline 4 & 0,971 & 5,11 & 98,854 & & & \\
\hline 5 & 0,218 & 1,146 & 100 & & & \\
\hline 6 & $4,01 \mathrm{E}-16$ & $2,11 \mathrm{E}-15$ & 100 & & & \\
\hline 7 & $3,02 E-16$ & 1,59E-15 & 100 & & & \\
\hline 8 & $2,25 \mathrm{E}-16$ & $1,18 \mathrm{E}-15$ & 100 & & & \\
\hline 9 & $1,76 \mathrm{E}-16$ & $9,27 E-16$ & 100 & & & \\
\hline 10 & $1,24 \mathrm{E}-16$ & $6,51 \mathrm{E}-16$ & 100 & & & \\
\hline 11 & $3,20 \mathrm{E}-17$ & 1,69E-16 & 100 & & & \\
\hline 12 & $2,71 \mathrm{E}-17$ & $1,43 \mathrm{E}-16$ & 100 & & & \\
\hline 13 & $-7,32 \mathrm{E}-18$ & $-3,86 \mathrm{E}-17$ & 100 & & & \\
\hline 14 & $-6,28 \mathrm{E}-17$ & $-3,31 \mathrm{E}-16$ & 100 & & & \\
\hline 15 & $-1,65 \mathrm{E}-16$ & $-8,67 \mathrm{E}-16$ & 100 & & & \\
\hline 16 & $-2,28 \mathrm{E}-16$ & $-1,20 \mathrm{E}-15$ & 100 & & & \\
\hline 17 & $-2,78 \mathrm{E}-16$ & $-1,47 \mathrm{E}-15$ & 100 & & & \\
\hline 18 & $-3,37 E-16$ & $-1,78 E-15$ & 100 & & & \\
\hline 19 & $-5,70 \mathrm{E}-16$ & $-3,00 \mathrm{E}-15$ & 100 & & & \\
\hline
\end{tabular}

Con base en estos resultados (leer porcentaje acumulado de las líneas 1, 2 y 3) se establece lo siguiente: el primer componente explica el $59,6 \%$ de los resultados, el segundo explica el $80,5 \%$ y el tercero explica el $93,7 \%$. Se procede entonces a asignar a cada variable evaluada un componente, de acuerdo al número mayor y como se observa en la tabla 4. El resultado de dicha asignación se observa en la tabla 5:

Tabla 4: Matriz de componentes principales

\begin{tabular}{|l|r|r|r|}
\hline \multirow{2}{*}{ Aspgen } & \multicolumn{3}{|c|}{ Componente } \\
\cline { 2 - 4 } & \multicolumn{1}{|c|}{1} & \multicolumn{1}{c|}{2} & \multicolumn{1}{c|}{3} \\
\hline Identificación &,- 784 &, 138 &, 561 \\
\hline Retención &, 907 &,- 368 &,- 138 \\
\hline Generación &, 503 &,- 423 &, 687 \\
\hline Compartir &, 303 &, 882 &, 254 \\
\hline Aplicación &,- 281 &, 481 &, 595 \\
\hline Tiposorg &, 959 &,- 422 &, 844 \\
\hline Cl &, 929 &, 098 &, 233 \\
\hline Estímulos &, 633 &,- 735 &, 314 \\
\hline Direccionan &, 974 &,- 127 &,- 018 \\
\hline Apropiación &, 821 &,- 567 &, 080 \\
\hline colaborativo &,- 836 &, 369 &, 014 \\
\hline websocial &,- 644 &, 407 &,- 4195 \\
\hline vigilancia &, 835 &,- 241 &,- 193 \\
\hline ciberaplic &,- 887 &, 196 &, 401 \\
\hline socializar &, 844 &, 507 &,- 019 \\
\hline exteriorizar &, 820 &, 562 &,- 078 \\
\hline Interiorizar &, 839 &, 532 &,- 027 \\
\hline Combinar &, 819 &, 531 &,- 031 \\
\hline
\end{tabular}


Tabla 5: Asignación de variables a factores

\begin{tabular}{|l|c|}
\hline \multicolumn{1}{|c|}{ Variable } & $\begin{array}{c}\text { Representada por el } \\
\text { factor de componente }\end{array}$ \\
\hline Aspgen & 3 \\
\hline Identificación & 1 \\
\hline Retención & 3 \\
\hline Generación & 2 \\
\hline Compartir & 3 \\
\hline Aplicación & 3 \\
\hline Tiposorg & 1 \\
\hline Cl & 1 \\
\hline Estímulos & 1 \\
\hline Direccionan & 1 \\
\hline Apropiación & 1 \\
\hline wcolaborativo & 2 \\
\hline websocial & 2 \\
\hline Vigilancia & 1 \\
\hline ciberaplic & 3 \\
\hline socializar & 1 \\
\hline exteriorizar & 1 \\
\hline Interiorizar & 1 \\
\hline Combinar & 1 \\
\hline
\end{tabular}

Posteriormente se da nombre a estos factores, así: Factor 1: Procesos de gestión conocimiento; Factor 2: Gestión de relaciones; y Factor 3: Trabajo colaborativo.

Con estos factores se hace la matriz de correlaciones para corroborar que efectivamente los componentes están bien agrupados (ver tabla 6). Los resultados son los mostrados en la Tabla 6. Los resultados muestran un nivel alto de significancia entre los nuevos 3 factores y ratifica que la gestión del conocimiento se está generando desde el desarrollo de procesos, la gestión de relaciones y el trabajo colaborativo.

Tabla 6: Correlaciones entre factores obtenidos

\begin{tabular}{|c|c|c|c|c|}
\hline \multicolumn{2}{|c|}{ Análisis } & $\begin{array}{l}\text { Puntuación de } \\
\text { factor } 1 \text { para }\end{array}$ & $\begin{array}{l}\text { Puntuación de } \\
\text { factor2para }\end{array}$ & $\begin{array}{l}\text { Puntuación de } \\
\text { factor3para } \\
\text { análisis } 1\end{array}$ \\
\hline \multirow{3}{*}{$\begin{array}{l}\text { Puntuación de factor1 para } \\
\text { análisis } 1\end{array}$} & Correlación de Pearson & 1 &, 000 &, 000 \\
\hline & Sig. (bilateral) & & 1,000 & 1,000 \\
\hline & $\mathrm{N}$ & 6 & 6 & 6 \\
\hline \multirow{3}{*}{$\begin{array}{l}\text { Puntuación de factor2para } \\
\text { análisis } 1\end{array}$} & Correlación de Pearson &, 000 & 1 &, 000 \\
\hline & Sig. (bilateral) & 1,000 & & 1,000 \\
\hline & $\mathrm{N}$ & 6 & 6 & 6 \\
\hline \multirow{3}{*}{$\begin{array}{l}\text { Puntuación de factor3para } \\
\text { análisis } 1\end{array}$} & Correlación de Pearson &, 000 &, 000 & 1 \\
\hline & Sig. (bilateral) & 1,000 & 1,000 & \\
\hline & $\mathrm{N}$ & 6 & 6 & 6 \\
\hline
\end{tabular}

\section{CONCLUSIONES}

De los resultados mostrados, de su análisis y de su discusión, se pueden obtener las siguientes conclusiones, sobre el análisis de capacidades de gestión del conocimiento para la competitividad de PYMES en Colombia:

El sector competitivo más afectado por el débil desarrollo de la gestión del conocimiento, es el del medio ambiente, situación que refleja lo que actualmente sucede en el país, pese a los esfuerzos, iniciativas, planes, programas y proyectos en este sector, parece ser que falta mucho más en el tema

Se nota un avance importante en lo relacionado con el sector de ciencia y tecnología, que viene reconociendo la importancia de la gestión del conocimiento en su desarrollo, pero igualmente como otros sectores, tiene mucho por mejorar, en especial con estímulos y apropiación de conocimiento. 
Es necesario que las empresas incorporen la gestión del conocimiento para innovar mediante el manejo direccionado de procesos de conocimiento (identificar, compartir, generar, retener y aplicar conocimiento), formando al capital humano en gestión de la información y la documentación, en gestión de la innovación y el cambio, en gestión del aprendizaje organizacional, en diseño de herramientas digitales y en gestión de la comunicación.

Se deben formular acciones para consolidar el uso de las TIC en función de gestión de información y conocimiento, la apropiación de herramientas de trabajo colaborativo, de gestión de proyectos, de gestión de relaciones sociales y de modelamiento y simulación.

También se deben proponer acciones para que las PYMES adopten prácticas de gestión del conocimiento como: sistemas de lecciones aprendidas, gestión de contenidos empresariales, trabajo en redes especializadas de información y conocimiento, sistemas de vigilancia tecnológica e inteligencia competitiva, entre otros.

\section{REFERENCIAS}

Alavy, M. y D.E. Leidner, Review knowledge management and knowledge management sytems conceptual foundatios and research issues, MIS Quarterly, ISSN: 2162-973 (en línea), 25(1), 107-136 (2001).http://www.jstor.org/discover/10.2307/3250961 ?uid=3737808\&uid=2\&uid=4\&sid=21102693099233.

Acceso: 6 de Julio (2012).

Álvarez, F.C., Riqueza natural de la nación. La gran paradoja, $1^{\text {a }}$ edición, 180-195, Torre Azul, Lima, Perú (2004).

Ampudia, M. L., Referentes teórico-conceptuales y desarrollo de redes de las Pymes en el contexto localregional-global. Revista de Ciencias Sociales y Humanidades, ISSN: 1669-1555 (en línea), 30 (15), 229256. (2006).http://www.redalyc.org/articulo.oa?id=85903009. Acceso 14 de Agosto (2012)

Andreu, R.A. y S. Siever, La gestión integral del conocimiento y del aprendizaje, Economía Industrial, ISSN: 0422-2784 (en línea), 326(2), 63-72 (1999).http://dialnet.unirioja.es/servlet/articulo?codigo=140115. Acceso: 2 de abril (2012)

Benavides, C.A., Tecnología, innovación y empresa, $1^{\underline{a}}$ edicion, Ediciones Piramide, Madrid, España(1998).

Bernal, C.A., A. Turriago y H.D. Sierra, Aproximación a la medición de la gestión del conocimiento, ADMINISTER, ISSN:1692-0279 (en línea), 16, 30-49

(2010).http://publicaciones.eafit.edu.co/index.php/administer/article/view/183/198\#.UkXmdtlagjY. Acceso: 11 de noviembre (2012).

Briceño, M.A. y C.A. Bernal, Estudios de caso sobre la gestión del conocimiento en cuatro organizaciones Colombianas líderes en penetración de mercado, Estudios gerenciales, ISSN 0123-5923 (en línea), 26(117), 173-193 (2010).http://www.icesi.edu.co/revistas/index.php/estudios_gerenciales/article/view/386. Acceso: 5 de febrero (2012).

Bueno, E., Enfoques principales y tendencias en dirección del conocimiento (Knowledgemanagement), en Dirección de Conocimiento: Desarrollo Teórico y aplicaciones por R. Hernández, ISBN: 84-88611-27-7 (en línea), 21-54, Ediciones La Coria, España (2003a).http://dialnet.unirioja.es/servlet/articulo?codigo=813647. Acceso: 4 de abril (2012)

Bueno, E., Gestión del Conocimiento: desarrollos teóricos y aplicaciones. 2ª edición, 25-38, Ediciones La Coria, Cáceres, España (2003b).

Cala, A., Situación y necesidades de la pequeña y mediana empresa, Civilizar, ISSN: 1657-8953 (en línea), 9, 1-22 (2005).http://www.redalyc.org/pdf/1002/100220350006.pdf. Acceso: 29 de septiembre (2012)

Carrillo, F.J., A global knowledge agenda based on capital systems (2005), http://www.knowledgesystems.org/Produccion_intelectual/capitulos/Global_Knowledge.pdf. Acceso: 3 de Mayo (2011).

CEN, Comité Européen de Normalisation, European Guide to good practice in knowledge management Part 1: Knowledge Management Framework, Bruselas, CEN (2004), 
http://cenftp1.cenorm.be/PUBLIC/CWAs/e-Europe/KM/CWA14924-01-2004-Mar.pdf. Acceso: 4 de Julio (2012).

Chen, T., A multiple-layer knowledge management system framework considering user knowledge privileges, International Journal of software engineering and knowledge engineering, issn: 0218-1940 (en línea), 19(3), 361-387 (2008).http://www.worldscientific.com/doi/abs/10.1142/S0218194009004192. Acceso 29 de Julio (2012)

Cuenca, L., A. Ortiz y A. Boza, Desarrollo de una Herramienta Software para la Vista de de la Arquitectura CIMOSA. Información Tecnológica, ISSN: $0718-0764$ (en línea), 19 (3), 97-106. (2008). http://www.scielo.cl/scielo.php?pid=S0718-07642008000300014\&script=sci_arttext. Acceso: 30 de Octubre (2012).

Davenport, T.H.yL. Prusak, Working knowledge: how organizations manage what they know, $2^{\mathrm{a}}$ edicion, Harvard Business School Press, United States of America (1998).http://www.kushima.org/is/wpcontent/uploads/2013/09/Davenport_know.pdf. Acceso 1 de Octubre (2012)

Del Moral, A., Gestión del conocimiento, 1a edición, 48-60, Thomson, Madrid, España (2007).

Díaz, Á., O. Lorenzo y L. Solís, Procesos de negocios de Pymes insertas en redes colaborativas. Revista Latinoamericana de Administración, ISSN: 1012-8255(en línea),34 (1), 25-46. (2005).http://www.redalyc.org/articulo.oa?id=71603403. Acceso 14 de Agosto (2012)

DNP, Departamento Nacional de Planeación, Cadenas productivas. Estructura, comercio internacional y protección, DNP (2005),

https://www.dnp.gov.co/Portals/0/archivos/documentos/DDE/PRESENTACION.pdf. Acceso: 22 de Noviembre (2011).

Domínguez, O., J. Finetti, R. Michel y B. Villanueva, Diseño de Sistemas de Procesos: Un Enfoque Integrador. Información Tecnológica, ISSN: 0718-0764 (en línea),18 (5), 11-20. (2007). http://www.scielo.cl/scielo.php?script=sci_arttext\&pid=S0718-

07642007000500003\&lng=es\&nrm=iso\&tlng=es. Acceso 14 de Agosto (2012)

Garzón, M.A., Aproximaciones a la gestión del conocimiento en empresas colombianas, Universidad Empresa, ISSN:0124-4639 (en línea),5(10), 232-256 (2006).http://revistas.urosario.edu.co/index.php/empresa/article/view/935. Acceso 9 de septiembre (2012).

Hautala, J., International academic knowledge creation and ba. A case study from Finland, Knowledge Management Research y Practice, ISSN:1477-8238 (en línea), 9, 4-16(2011). http://www.docstoc.com/docs/134321390/Article17-International-academic-knowledge-creation. Acceso 15 de Octubre (2012).

Johannessen, J.A., B. Olsena y J. Olaisen, Intellectual capital as a holistic management philosophy: a theoretical perspective, International Journal of Information Management, ISSN: 0378-7206 (en línea), 25(2), 151-171 (2005).http://www.sciencedirect.com.ezproxy.unal.edu.co/science/article/pii/S0268401204001367. Acceso 3 de Agosto (2012).

Knudsen, C., Theories of the firm, strategic management, and leadership, resource-based an evolutionary theories of the firm: Towards a Synthesis, 1a edición, Kluwer Academic Publishers, ISSN:0925-5001 (en línea), 179-203, Londres, (1995).

http://www.sciencedirect.com.ezproxy.unal.edu.co/science/article/pii/S0268401204001367. Acceso: 15 de abril (2012)

Kok, A., Intellectual capital management as part of knowledge. management initiatives at institutions of higher learning, The Journal of Knowledge Management, ISSN: 1479-4411 (en línea), 5(2), 181-192 (2007). http://www.ejkm.com/volume5/issue2. Acceso 16 de Marzo (2012).

Lobato, V., Caracterización del conocimiento en PYMES que realizan proyectos TIC: un modelo de análisis y valoración, Tesis de doctorado, Departamento de explotación y prospección de minas, Universidad de Oviedo, Oviedo, España (2008).https://www.educacion.gob.es/teseo/imprimirFicheroTesis.do?fichero=9555. Acceso: 1 de mayo (2012). 
López, M. y G. Calderón, Análisis de las dinámicas culturales al interior de un clúster empresarial, Estudios gerenciales, ISSN: 0123-5923, 99, 13-37 (2006).http://www.scielo.org.co/pdf/eg/v22n99/v22n99a01.pdf. Acceso 8 de Mayo (2012)

López, M., Ciudadanía Digital, un modelo de implantación en la región de Manizales y Caldas, Tesis de doctorado, Departamento de Ingeniería, Universidad Pontificia de Salamanca, Madrid, España (2010).http://gisti.upsam.es/index.php/tesisdoctoral. Acceso 12 de Julio (2012).

López, M., C.E. Marulanda y G.A. Isaza, Cultura organizacional y gestión del cambio y de conocimiento en las organizaciones de Caldas, Revista Escuela de Administración de Negocios, ISSN: 0120-8160 (en línea), 33, 117-139 (2011).http://www.scielo.org.co/scielo.php?script=sci_arttext\&pid=S0120$81602012000100006 \&$ Ing=pt\&nrm=iso. Acceso: 23 de Junio (2012).

Marr, B., Measuring and benchmarking intellectual capital, Benchmarking: An International Journal, ISSN:1463-5771 (en línea), 11(6), 559-570 (2004).

http://www.emeraldinsight.com/journals.htm?articleid=843125. Acceso 22 de Junio (2012).

Martínez, M.R,Desarrollo de un modelo de gestión del conocimiento en la cadena de suministro de la industria agroalimentaria, Tesis de doctorado, Departamento de Ingeniería de Organización, Universidad Politécnica de Madrid, Madrid, España (2011).http://oa.upm.es/6199/. Acceso 8 de Septiembre (2012).

Martínez, M.R., A procedure to design a structural and measurement model of intellectual capital: An exploratory study, Information and management, ISSN:0378-7206 (en línea), 43 (5), 617-626, (2006). http://www.sciencedirect.com.ezproxy.unal.edu.co/science/article/pii/S0378720606000310. Acceso 4 de diciembre (2012).

Matilla, M.M. y R. Chalmeta, Metodología para la Implantación de un sistema de medición del rendimiento Empresarial, Información Tecnológica, ISSN: 0718-0764 (en línea), 18(1), 119-126 (2007).http://www.scielo.cl/pdf/infotec/v18n1/art16.pdf. Acceso: 17 de Febrero (2012).

Meisel, C.A., H.P. Bermeo y L. Oviedo, Generación de valor a través de la gestión estratégica del conocimiento, de la innovación y la mejora continua "Un modelo de aplicación al sistema de operaciones de una PYME manufacturera", Scientia et Technica, ISSN:0122-1701 (en línea), 12(31), 165-170 (2006). http://www.redalyc.org/articulo.oa?id=84911639029. Acceso: 10 de Marzo (2012).

Morales, M.E., S.E. Sanabria y M.A. Arias, Acumulación de conocimiento, innovación y competitividad en aglomeraciones empresariales, Revista Facultad de Ciencias Económicas, ISSN:0121-6805 (en línea), 18(2), 19-53 (2010).http://www.scielo.org.co/scielo.php?pid=S0121-68052010000200003\&script=sci_arttext. Acceso: 26 de Julio (2012).

Muñoz, D., y D. Muñoz, Planeación y Control de Proyectos con Diferentes Tipos de Precedencias Utilizando Simulación Estocástica. Información Tecnológica, ISSN: 0718-0764 (en línea), 21(4), 25-33 (2010). http://www.scielo.cl/scielo.php?script=sci_arttext\&pid=S0718-

07642010000400005\&lng=es\&nrm=iso\&tlng=es. Acceso 13 de Agosto (2012).

Nahapiet, J. y S. Ghoshal, Social capital, intellectual capital, and the organizational advantage, The Academy of Management Review, ISSN:0363-7425 (en línea), 23(2), 242-266 (1998).http://www.jstor.org/discover/10.2307/259373?uid=3737808\&uid=2\&uid=4\&sid=21102693294323.

Acceso: 19 de Febrero (2012).

OCDE, Organisation for Economic Co-operation and Development, Industrial Competitiveness, 1a edición, 75-89, OCDE, París, Francia (1997).

OCDE, Organisation for Economic Cooperation and Development, Manual de Oslo: Guía para la recogida e interpretación de datos sobre innovación. 3aㅡ edición, 101-142, Grupo Tragsa, París, Francia (2006).

Paniagua, E., y otros 14 autores, Fundamentos de la Gestión Tecnológica del Conocimiento, en La Gestión Tecnológica del Conocimiento, por Paniagua, E., 1aㅡ edición, 12- 82, Servicio de publicaciones Universidad de Murcia, Murcia, España (2007)

Porter, M.E., La ventaja competitiva de las naciones, 1르 edición, 48-57, Vergara, Buenos Aires, Argentina (1991). 
Romero, D.O., L.M. Mathison y D. Rojas, Una propuesta de gestión de conocimiento para la pequeña y mediana empresas, Revista de Micro y Pequeña Empresa, ISSN: 2011-9097(en línea), 3(1), 120-143 (2009).http://core.kmi.open.ac.uk/display/5753273. Acceso 14 de Abril (2012).

Sánchez, J.J., J. Osorio y E. Baena, Algunas aproximaciones al problema de financiamiento de las PYMES de Colombia, Scientia et Technica, ISSN: 0122-1701 (en línea), 13(34), 321-324 (2007).http://repositorio.utp.edu.co/dspace/bitstream/11059/180/1/aproximaciones-problema-financiamientopymes.pdf. Acceso: 19 de Agosto (2012).

Shin, M., T. Holdeny R.A. Schmidt, From knowledge theory to management practice: towards an integrated approach, Information Processing and Management, ISSN:0306-4573 (en línea), 37(2), 335-355 (2001).http://www.sciencedirect.com.ezproxy.unal.edu.co/science/article/pii/S0306457300000315. Acceso 12 de Noviembre (2012). 
